\title{
SYNTHESIS AND PHOTOLUMINESCENCE STUDIES ON ZINC OXIDE NANOWIRES
}

\author{
Nguyen Ngoc Long*, Ngo Xuan Dai, Nguyen Thi Thuc Hien \\ Center for Materials Science, Hanoi University of Science, \\ Vietnam National University, Hanoi, \\ 334 Nguyen Trai Rd., Thanh Xuan Dist., Hanoi, Vietnam
}

Received 27 November 2006

\begin{abstract}
Semiconductor single crystal $\mathrm{ZnO}$ nanowires have been successfully synthesized by a simple method based on thermal evaporation of $\mathrm{ZnO}$ powders mixed with graphite. Metallic catalysts, carrying gases, and vacuum conditions are not necessary. The x-ray diffraction (XRD) analysis shows that the $\mathrm{ZnO}$ nanowires are highly crystallized and have a typical wurtzite hexagonal structure with lattice constants $\mathrm{a}=0.3246 \mathrm{~nm}$ and $\mathrm{c}=0.5203 \mathrm{~nm}$. The scanning electron microscopy (SEM) images of nanowires indicate that diameters of the $\mathrm{ZnO}$ nanowires normally range from 100 to $300 \mathrm{~nm}$ and their lengths are several tens of micrometers. Photoluminescence (PL) and photoluminescence excitation (PLE) spectra of the nanowires were measured in the range of temperature from $15 \mathrm{~K}$ to the room temperature. Photoluminescence spectra at low temperatures exhibit a group of ultraviolet (UV) narrow peaks in the region $368 \mathrm{~nm} \sim 390 \mathrm{~nm}$, and a blue-green very broad peak at $500 \mathrm{~nm}$. Origin of the emission lines in PL spectra and the lines in PLE spectra is discussed.
\end{abstract}

Keywords: Semiconductors, nanostructures, photoluminescence, exciton, zinc oxide

\section{INTRODUCTION}

In recent years, one-dimensional (1D) semiconductor nanostructures including nanowires, nanorods, nanobelts and nanotubes have attracted much attention due to their importance in basic scientific research and potential technology applications. It is generally accepted that the 1D nanostructures are useful materials for investigating the dependence of electrical and thermal transport or mechanical properties on dimensionality and size reduction (or quantum confinement). They are also expected to play an important role as both interconnects and functional units in fabricating electronic, optoelectronic, electrochemical and electromechanical nanodevices [1]. In the past few years, much effort has been devoted to developing various 1D semiconductor nanostructures. Many methods have been used to synthesize various metal oxides, III-V and II-VI compound semiconductor nanostructures: vapor-phase transport [2, 3], laser ablation [4], chemical vapor deposition [5], metal-organic chemical vapor deposition [6, 7], and hydrothermal method [8].

Zinc oxide $(\mathrm{ZnO})$ is recognized as a promising material for photonics because of its wide bandgap $E_{\mathrm{g}}$ of $3.37 \mathrm{eV}$ and large exciton binding energy of $60 \mathrm{meV}$. Furthermore, $\mathrm{ZnO}$ is bio-safe and biocompatible, and may be used for biomedical applications without coating. Various kinds of $\mathrm{ZnO}$ nanostructures have been realized, such as nanowires, nanorods, nanobelts, nanotubes

\footnotetext{
*Corresponding author e-mail: longnn@vnu.edu.vn
} 
etc. [9]. Among the 1D nanostructures, $\mathrm{ZnO}$ nanowires and nanorods have been widely studied because of their easy nanomaterials formation and device applications.

In this paper, we present a metallic catalyst-free growth of $\mathrm{ZnO}$ nanowires using a simple method based on thermal evaporation of $\mathrm{ZnO}$ powders mixed with graphite. Photoluminescence (PL) and photoluminescence excitation (PLE) spectra of the nanowires were measured in the range of temperature from $15 \mathrm{~K}$ to the room temperature. PL spectra are characterized by emissions from different mechanisms including free exciton $(\mathrm{X})$, neutral donor-bound exciton $\left(\mathrm{D}^{\circ} \mathrm{X}\right)$, recombination of an electron bound on a shallow donor with a free hole in the valence band (BF) [10], transition related to deep defects. It is found that the UV emission at room temperature is a mixture of emission due to free exciton and emission concerned with BF transition. Especially, the low temperature PLE spectra exhibit a fine structure related to the A and $\mathrm{B}$ exciton and other band edge transitions.

\section{EXPERIMENTS DETAILS}

Nanowires of $\mathrm{ZnO}$ were synthesized by thermal evaporation of a mixture of $\mathrm{ZnO}$ and graphite powders with molar ratio ranged from 1:2 to 1:4. The source material was placed at the closed end of a quartz tube. The other end of the quartz tube was open to the atmosphere. The quartz tube was inserted into a horizontal tube furnace, so that the source material was placed in the temperature region approximately $1050^{\circ} \mathrm{C}$. The $\mathrm{SiO}_{2} / \mathrm{Si}$ substrates were placed along the quartz tube in the temperature regions from $900^{\circ} \mathrm{C}$ to $650^{\circ} \mathrm{C}$. After $45 \mathrm{~min}$ evaporation, the quartz tube was drawn out from the furnace and cooled down to room temperature. White color films were formed on the $\mathrm{SiO}_{2} / \mathrm{Si}$ substrates. The crystal structure of the films was analyzed using a SIMENS D5005 X-ray diffractometer. The morphology of the films was characterized using a scanning electron microscope (JEOL $5410 \mathrm{LV}$ ). The composition of the films was determined by an energy dispersive X-ray (EDX) spectrometer (EDS, OXFORD ISIS 300) attached to the JEOL-JSM 5410 LV scanning electron microscope. PL and PLE spectra were measured at temperatures ranging from $15 \mathrm{~K}$ up to $300 \mathrm{~K}$ using a Fluorolog FL3-22 Jobin Yvon Spex USA spectrofluorometer with a xenon lamp of $450 \mathrm{~W}$ as an excitation source.

\section{RESULTS AND DISCUSSION}

Figure 1 shows typical scanning electron microscope (SEM) images of $\mathrm{ZnO}$ nanowires. The diameters of $\mathrm{ZnO}$ nanowires normally range from 100 to $300 \mathrm{~nm}$ and their lengths are several tens of micrometers.

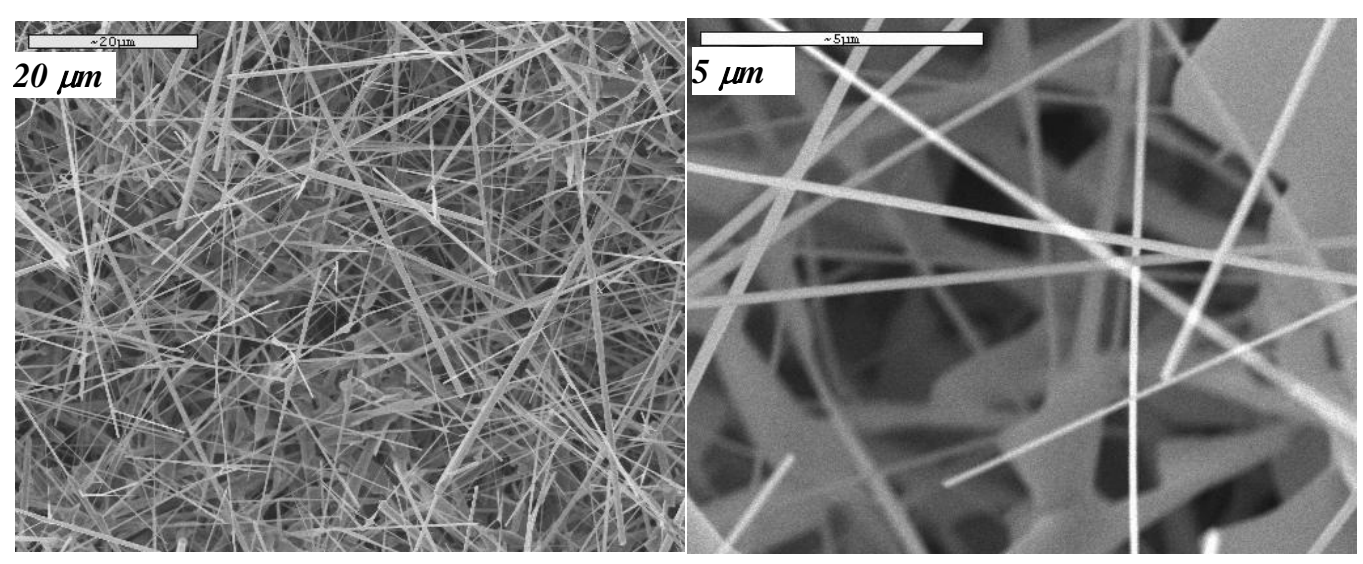

Fig. 1. SEM images of $\mathrm{ZnO}$ nanowires 
An X-ray diffraction (XRD) pattern of $\mathrm{ZnO}$ bulk nanowires is shown in Fig. 2. The XRD pattern clearly indicates that the $\mathrm{ZnO}$ nanowires possess a hexagonal wurtzite crystal structure with lattice constants of $a=0.3246 \mathrm{~nm}$ and $c=0.5203 \mathrm{~nm}$. The (002) diffraction peak is dominant in the spectrum, which means that the nanowires have preferential growth direction along $c$-axis.

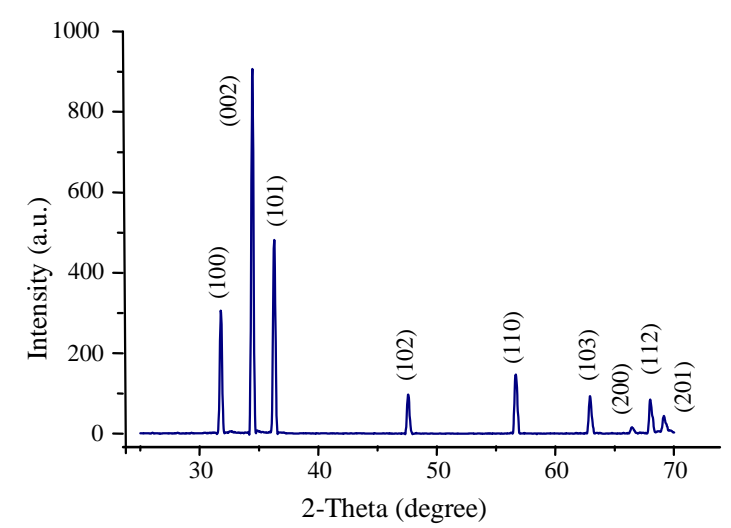

Fig. 2: XRD pattern of $\mathrm{ZnO}$ nanowires

EDX measurements were carried out in order to examine the elemental composition of the $\mathrm{ZnO}$ nanowires. A typical EDX spectrum of the $\mathrm{ZnO}$ nanowires is shown in Fig. 3. It can be seen from the figure, the nanowires are composed of zinc and oxygen.

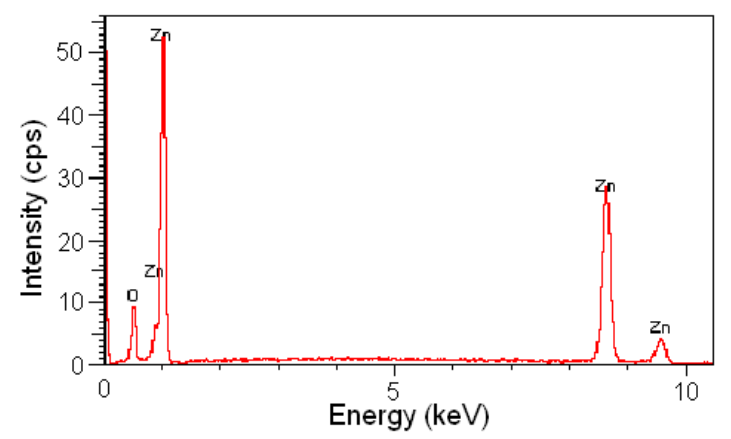

Fig. 3: EDX spectrum of $\mathrm{ZnO}$ nanowires

PL spectrum of $\mathrm{ZnO}$ nanowires measured at $30 \mathrm{~K}$ using wavelength of $335 \mathrm{~nm}$ of a xenon lamp of $450 \mathrm{~W}$ as an excitation source is shown in Fig. 4. Photoluminescence spectra at low temperatures exhibit a group of ultraviolet (UV) narrow peaks in the region $368 \mathrm{~nm} \sim 390 \mathrm{~nm}$ $(3.179 \mathrm{eV} \sim 3.369 \mathrm{eV})$, and a blue-green very broad peak at $500 \mathrm{~nm}(2.480 \mathrm{eV})$.

This blue-green broad peak is found to have a distinct structure due to multi-phonon emission. The phonon energy on the average is about $70 \mathrm{meV}$, which is approximately equal to the energy of LO-phonon in $\mathrm{ZnO}(\sim 72 \mathrm{meV})$. The blue-green broad peak is usually associated with deep defects such as singly ionized oxygen vacancies [11] or copper-related defects [12]. In this paper, we focus our attention only on the peak group in the UV region, concerning the origin of these emission peaks.

In order to investigate the origins of emission peaks, the PL spectra were measured in the temperature range from $15 \mathrm{~K}$ to $300 \mathrm{~K}$. The PL spectrum at $15 \mathrm{~K}$ exhibits five emission peaks at 
3.362, 3.314, 3.296, 3.240, and 3.223 eV (Fig. 5). The peak at $3.362 \mathrm{eV}$ with full width at halfmaximum value of $16 \mathrm{meV}$, as will be shown later, is attributed to neutral donor-bound exciton $\left(\mathrm{D}^{\circ} \mathrm{X}\right)$, the peak at $3.314 \mathrm{eV}$ is attributed to recombination of an electron bound on a shallow donor with a free hole in the valence band (BF). The peaks at 3.296 and $3.223 \mathrm{eV}$ are interpreted as phonon replicas of $\mathrm{D}^{\circ} \mathrm{X}$ with one and two LO-phonon energy of $72 \mathrm{meV}\left(\mathrm{D}^{\circ} \mathrm{X}-1 \mathrm{LO}, \mathrm{D}^{\circ} \mathrm{X}-\right.$ $2 \mathrm{LO})$, the peak at $3.240 \mathrm{eV}$ is interpreted as a phonon replica of $\mathrm{BF}(\mathrm{BF}-1 \mathrm{LO})$.

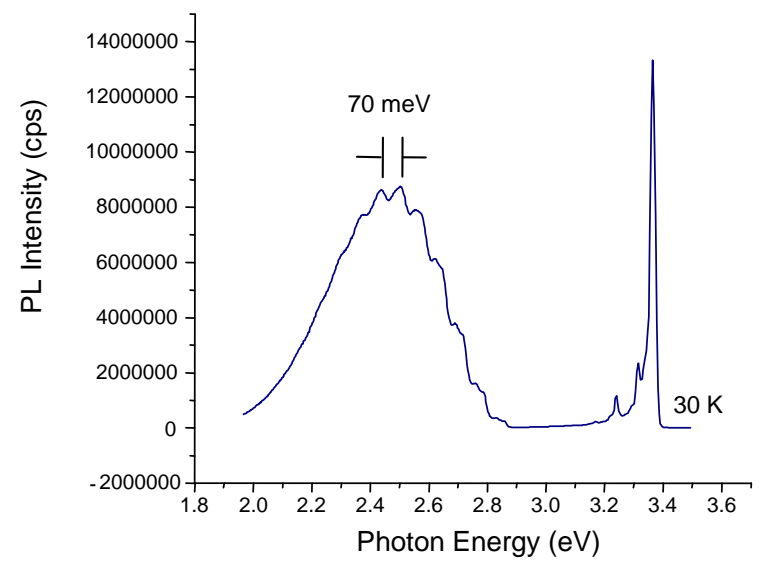

Fig. 4: PL spectrum of $\mathrm{ZnO}$ nanowires measured at $30 \mathrm{~K}$ using wavelength of $335 \mathrm{~nm}$ of a xenon lamp of $450 \mathrm{~W}$ as an excitation source

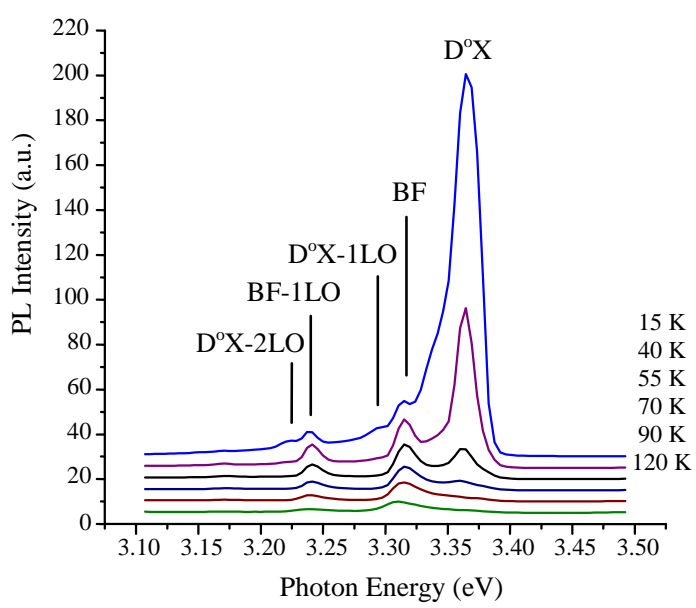

Fig. 5: Low temperature PL spectra of ZnO nanowires measured at various temperatures. Here only UV peak group is shown

As it can be seen from Fig. 5 and Fig. 6, the intensity of the sharp peak at $3.362 \mathrm{eV}\left(\mathrm{D}^{\circ} \mathrm{X}\right)$ is decreased rapidly and its position is shifted slightly to the low-energy side with increasing measuring temperature. At $120 \mathrm{~K}$, this peak becomes much weaker than the BF peak at $3.314 \mathrm{eV}$.

From Fig. 6 it is also evident that beginning from $70 \mathrm{~K}$ a shoulder with an energy value of 3.377 $\mathrm{eV}$ appears at the high-energy side of the $\mathrm{D}^{\circ} \mathrm{X}$ peak. The position of the shoulder is shifted to the low-energy side with increasing measuring temperature. This shoulder is still maintained up 
to $250 \mathrm{~K}$ and is mixed to the BF peak at higher temperatures. This shoulder is interpreted to be the origin from the radiative recombination of free exciton $\left(\mathrm{X}_{\mathrm{A}}\right)$. Indeed, as temperature is increased, the thermal activation energy is enough for the release of excitons from the neutral donor $\left(\mathrm{D}^{\circ} \mathrm{X} \rightarrow \mathrm{D}^{\circ}+\mathrm{X}\right)$, then, radiative transitions take place via states of the free excitons.

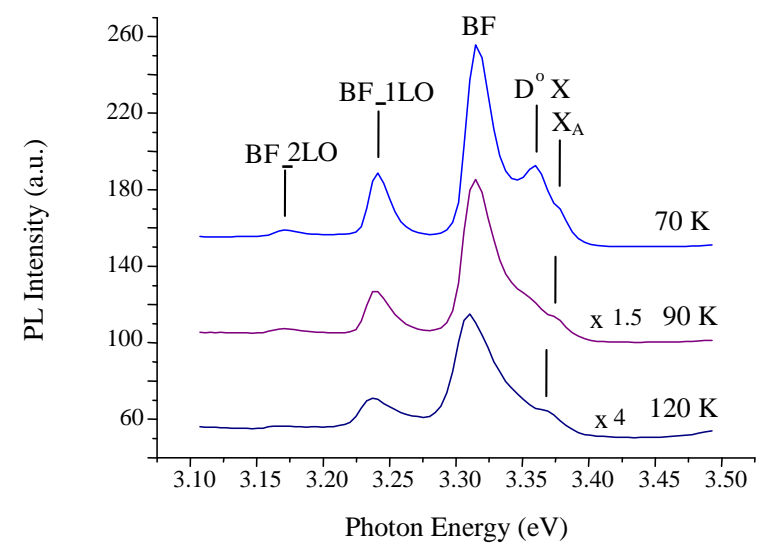

Fig. 6: PL spectra of ZnO nanowires at 70, 90, and $120 \mathrm{~K}$. The spectra exhibit shoulders due to emission of free exciton $A\left(X_{A}\right)$

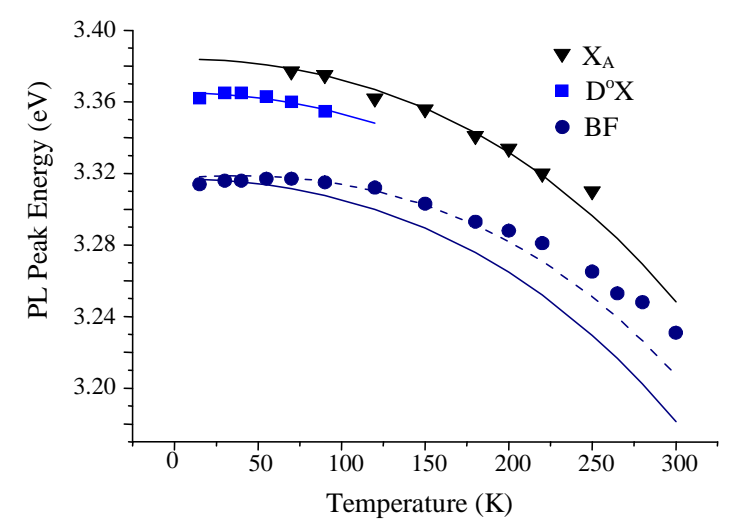

Fig. 7: The temperature dependence of the peak energies for $X_{A}$ emission (solid triangle), $D^{o} X$ emission (solid squares), and BF emission (solid circle)

Assuming that the peak position of free exciton and donor-bound exciton emission varies with temperature as the energy band gap, we tried to fit the observed temperature dependence to Varshni's semiempirical formula [13]:

$$
E(T)=E(0)-\alpha T^{2} /(T+\beta)
$$

where $E(0), \alpha$ and $\beta$ are fitting parameters. As it can be seen in Fig. 7, the experimental values for the $\mathrm{X}_{\mathrm{A}}$ peak and the $\mathrm{D}^{\circ} \mathrm{X}$ peak fit rather well to the Varshni's curve (solid lines in the Fig. 7) with fitting parameters: $\alpha=-9.8 \times 10^{-4} \mathrm{eV} / \mathrm{K}, \beta=-950 \mathrm{~K}$, and $E(0)=3.384 \mathrm{eV}$ and $3.365 \mathrm{eV}$ for $\mathrm{X}_{\mathrm{A}}$, and $\mathrm{D}^{\circ} \mathrm{X}$ peaks, respectively. 
The binding energy of the exciton at neutral donor is estimated to be about $19 \mathrm{meV}$ in accordance with the results in [14]. If we recognize a linear relation between the binding energy of the exciton at neutral donor $E_{\text {bind. } D^{o} X}$ and the binding energy of the donor $E_{D}$ like in [14]:

$$
E_{\text {bind } . D^{o} X}=A+B \cdot E_{D}
$$

with constants $A=-3.8 \mathrm{meV}$ and $B=0.375$, we can estimate $E_{D}=62.5 \mathrm{meV}$.

For the origin of the BF emission peak at $3.314 \mathrm{eV}$, we believe that this peak probably corresponds to the recombination of a free carrier with a carrier bound on a defect. In our case, the sample is an n-type semiconductor, so it is more likely that an electron bound on a donor recombines with a free hole in the valence band $(\mathrm{BF})$. In that case, the peak position of the BF emission should vary with temperature more slowly than the energy band gap does according to Varshni's model, as may be seen in Fig. 7. The experimental values of the BF peak position at various temperatures fit rather well to the curve (dotted line in the Fig. 7) calculated using the formula described the peak position of emission due to transitions from a donor to the valence band [15]:

$$
h v=E_{g}-E_{D}+k_{B} T
$$

where $E_{g}$ is the band-gap energy, $E_{D}$ as mentioned above, is the binding energy of the donor,

$T$ is temperature, $k_{B}$ is Boltzmann constant. The free-to-bound radiative transitions have been observed in $\mathrm{ZnO}$ both at low temperatures and at room temperature by other authors [16, 17].

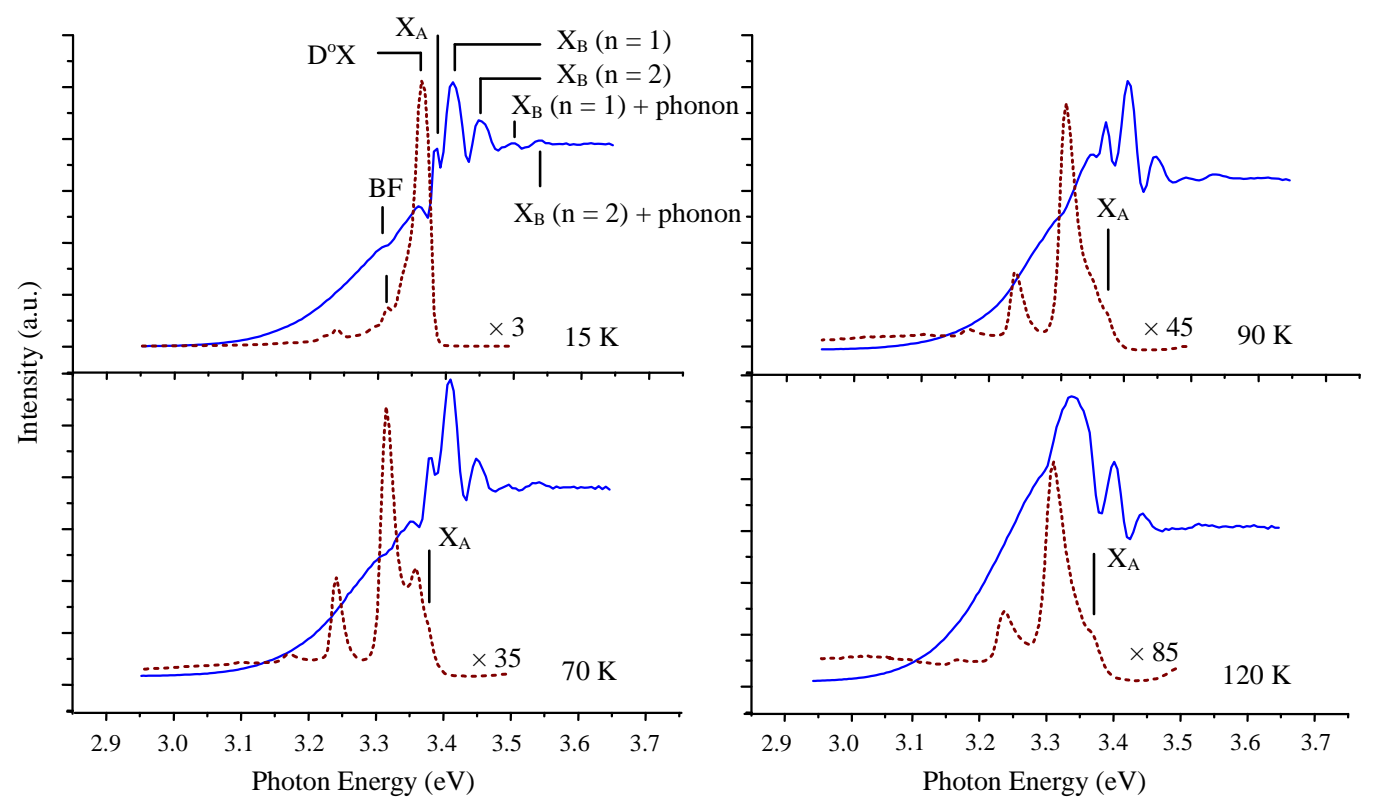

Fig. 8: The PLE (solid lines) and PL (dotted lines) spectra of ZnO nanowires measured at different temperatures. In the figure are shown the emission peaks related to the free exciton $\left(X_{A}, X_{B}\right)$, the neutral donor-bound exciton $\left(D^{o} X\right)$ and the bound-to-free transitions $(B F)$

Photoluminescence excitation (PLE) spectra for the broad emission peak at $495 \mathrm{~nm}(2.505 \mathrm{eV})$ were measured in the range of temperatures from $15 \mathrm{~K}$ to $300 \mathrm{~K}$. The PLE spectra at low 
temperatures exhibit fine structures (Fig. 8). The PLE spectrum at $15 \mathrm{~K}$ consists of a weak shoulder at $3.309 \mathrm{eV}$ and six peaks at 3.361, 3.386, 3.411, 3.453, 3.499, and $3.538 \mathrm{eV}$. These peaks are shifted slightly to the low-energy side with increasing measuring temperature.

In order to find the origins of the PLE peaks, we compare the PLE spectra with the PL spectra measured at the same temperatures (Fig. 8). It is evident from Fig. 8 that the shoulder at 3.309 $\mathrm{eV}$ can be attributed to absorption transition from the valence band to a donor (BF). The peak at $3.361 \mathrm{eV}$ is due to absorption transition of neutral donor-bound exciton $\left(\mathrm{D}^{\circ} \mathrm{X}\right)$. The peak at $3.386 \mathrm{eV}$ is attributed to absorption transition of the A-free exciton $\left(\mathrm{X}_{\mathrm{A}}\right)$. The peaks at 3.411 and $3.453 \mathrm{eV}$ are interpreted as absorption transitions to the ground $(\mathrm{n}=1)$ and the first excited $(\mathrm{n}=$ 2) states of the $B$-free exciton $\left(X_{B}\right)$, respectively.

The energy separation between the $n=1$ and $n=2$ states is $42 \mathrm{meV}$. Assuming the hydrogenic model based on the effective mass approximation is applicable, the binding energy of the Bexciton estimated from the separation of $42 \mathrm{meV}$ is $56 \mathrm{meV}$ in good accordance with the results in [18].

The peak at 3.499 and $3.538 \mathrm{eV}$ can be attributed to absorption transitions to states $\mathrm{n}=1,2$ of the B-exciton accompanied by an absorption of phonon $\left(\mathrm{X}_{\mathrm{B}}(\mathrm{n}=1)+\right.$ phonon, $\mathrm{X}_{\mathrm{B}}(\mathrm{n}=2)+$ phonon), respectively.

\section{CONCLUSIONS}

The $\mathrm{ZnO}$ nanowires with diameters ranging from 100 to $300 \mathrm{~nm}$ and lengths of several tens of micrometers have been successfully prepared by thermal evaporation method without metallic catalyst. The $\mathrm{ZnO}$ nanowires possess a hexagonal wurtzite crystal structure with lattice constants of $a=0.3246 \mathrm{~nm}$ and $c=0.5203 \mathrm{~nm}$. The A-free exciton $\left(\mathrm{X}_{\mathrm{A}}\right)$, the neutral donorbound exciton $\left(\mathrm{D}^{\circ} \mathrm{X}\right)$ and the bound-to-free $(\mathrm{BF})$ transition were observed both in low temperature PL spectra and in PLE spectra. Absorption transitions related to the ground and the first-excited states of the B-exciton $\left(\mathrm{X}_{\mathrm{B}}\right)$ were observed in the PLE spectra measured at low temperatures. The room temperature PL in the UV region is a mixture of emission due to free excition and emission concerned with transitions from a donor level to the valence band.

\section{ACKNOWLEDGEMENT}

This work was supported by Natural Science Council, Ministry of Science and Technology of Vietnam (Project 811304).

\section{REFERENCES}

1. Xia, Y.N., Yang, P.D., Sun, Y.G., Wu, Y.Y., Mayers, B., Gates, B., Yin, Y.D., Kim, F., and Yan, H.Q. (2003), Adv. Mater., vol. 15, No. 5, pp. 353-389.

2. Huang, M., Mao, S., Feick, H., Yan, H., Wu, Y., Kind, H., Weber, E., Russo, R., and Yang, P. (2001), Science, vol. 292, pp. 1897-1899.

3. Pan, Z.W., Dai, Z.R., and Wang, Z.L. (2001), Science, vol. 291, pp. 1947-1949.

4. Morales, A.M. and Lieber, C.M. (1998), Science, vol. 279, pp. 208-211.

5. Najafov, H., Fukada, Y., Ohshio, S., Iida, S., and Saitoh, H. (2003), Jpn. J. Appl. Phys., vol. 42, No. 6A, pp. 3490-3495.

6. Park, W.I., Kim, D.H., Jung, S.W., and Yi, G.C. (2002), Appl. Phys. Lett., vol. 80, No. 22, pp. 4232-4234. 
7. Zhang, B.P., Binh, N.T., Segawa, Y., Wakatsuki, K., and Usami, N. (2003), Appl. Phys. Lett., vol. 83, No. 8, pp. 1635-1637.

8. Min Guo, Peng Diao, Shengmin Cai (2005), Applied Surface Science, vol. 249, pp. 71-75.

9. Wang, Z.L. (2004), Mater. Today, vol. 7, No. 6, pp. 26-34.

10. Dam Hieu Chi, Le Thi Thanh Binh, Nguyen Thanh Binh, Le Duy Khanh, Nguyen Ngoc Long (2006), Applied Surface Science, vol. 252, No. 8, pp. 2770-2775.

11. Vanhuesden, K., Warren, W.L., Seager, C.H., Tallant, D.R., Voigt ,J.A., and Gnade, B.E. (1996), J. Appl. Phys., vol. 79, No. 10, pp. 7983-7990.

12. Dingle, R. (1969), Phys. Rev. Lett., vol. 23, No. 11, pp. 579-581.

13. Varshni, Y.P. (1967), Physica (Amsterdam), vol. 34, No. 1, pp. 149-154.

14. Meyer, B.K., Sann, J., Hofmann, D.M., Neumann, C., and Zeuner, A. (2005), Semicond. Sci. Technol., vol. 20, pp. S62-S66.

15. Dean, P.J. (1973), Progress in Solid State Chemistry, vol. 8, pp. 1-126.

16. Zhang, B.P., Binh, N.T., Wakatsuki, K., Segawa, Y., Kashiwaba, Y., and Haga, K. (2004), Nanotechnology, vol. 15, pp. S382-S388.

17. Zhao, Q.X., Willander, M., Morjan, R.E., Hu, Q.H., and Campbell, E.E.B. (2003), Appl. Phys. Lett., vol. 83, No. 1, pp. 165-167.

18. Shan, W., Walukiewicz, W., Ager, III J.W., Yu, K.M., Yuan, H.B., Xin, H.P., Cantwell, G., and Song, J.J. (2005), Appl. Phys. Lett., vol. 86, p. 191911. 